\title{
Cross-sectional interventional radiology report
}

Accepted: 18 November 2020

(c) Springer Science+Business Media, LLC, part of Springer Nature 2021
The COVID-19 pandemic created a lot of uncertainty with regard to its impact on radiology services, including interventional procedures. The CSIR ETC put out a statement, published in the American Journal of Roentgenology, "Reorganizing Cross-Sectional Interventional Procedures Practice during the Coronavirus Disease (COVID-19) Pandemic," [1] to coalesce insights gleaned from the experience of members of the CSIR ETC.

The CSIR ETC has also been active on social media, using Twitter as its primary platform, under the handle: @CsirEtc. A dedicated web page was also developed on the SAR website, with the mission, goals, and membership of the CSIR ETC.

\section{Look ahead}

The year ahead has already been active, with numerous initiatives by the CSIR ETC and its members. We are performing a review on post-procedure observation times as well as publishing our own recommendations based on this review and our experience. Additionally, the use of personal protective equipment is quite variable among institutions, and we are looking to analyze our experience and outcomes with regard to infection rates. We are also working on a statement on the current and future state of procedures within abdominal radiology. Additionally, the CSIR ETC is working on an upcoming special section in Abdominal Radiology related to procedural care. Finally, the CSIR ETC is working on both hands-on and didactic workshops for the upcoming SAR annual meeting in 2021.

\section{References}

1. Fananapazir G, Lubner MG, Mendiratta-Lala M, WildmanTobriner B, Galgano SJ, Lamba R, Hinshaw JL, Brook OR. Reorganizing Cross-Sectional Interventional Procedures Practice During the Coronavirus Disease (COVID-19) Pandemic. American Journal of Roentgenology. 2020 Apr 25:1-5.

Publisher's Note Springer Nature remains neutral with regard to jurisdictional claims in published maps and institutional affiliations. 